\title{
Ökologische Untersuchungen zur Nitrifikation in Nord-und Ostsee
}

\author{
Gerhard RHEINHEIMER \\ Institut für Meereskunde der Universität Kiel, Kiel
}

\begin{abstract}
Ecological investigations on nitrification in North and Baltic Sea. Ammonia, nitrite and nitrate were regularly estimated at several stations in the Kieler Bucht (western Baltic Sea) since November 1964. There are considerable seasonal changes in the contents of these 3 nitrogen compounds with impressive maxima of nitrite and nitrate in February or at the beginning of March. The great increase of nitrite and nitrate during the winter and also a smaller increase in summer are mainly caused by oxidation of ammonia, first to nitrite and then to nitrate, by nitrifying bacteria. In consequence chemoautotrophic nitrite- and nitratebacteria could be found in the water as well as in sediments all over the Kieler Bucht and also in the North Sea around the isle of Helgoland. These nitrifying bacteria are able to oxidize ammonia or nitrite in salinity conditions typical for the western Baltic Sea and the North Sea.
\end{abstract}

\section{EINLEITUNG}

In den Meeren liegt die Hauptmenge des gebundenen Stickstoffs in Form von Nitrat vor. Nach Vaccaro (1962) soll dessen Anteil etwa 65\% betragen. Da der organisch - das heißt in erster Linie von den verschiedenen Lebewesen - gebundene Stickstoff aber immer wieder fast ausschließlich in Form von Ammoniak freigesetzt wird, muß also ständig Ammoniak über Nitrit zu Nitrat oxydiert werden. Während man über diese sogenannte Nitrifikation im Erdboden und in den Binnengewässern schon recht gut Bescheid weiß (ENGEL 1958, 1960), ist über deren Ablauf im Meer noch kaum etwas bekannt. Nach ZoBell (1946) müssen auch in den Meeren in erster Linie Bakterien für die Ammoniakoxydation verantwortlich sein, denn die photochemische Oxydation bleibt auf die obersten Zentimeter des Wasserkörpers beschränkt und eine rein chemische $\mathrm{NH}_{4}+$-Oxydation ließ sich unter den im Meere gegebenen Bedingungen nicht feststellen. Trotz zahlreicher Untersuchungen konnten nitrifizierende Bakterien aber bis vor kurzem nur in Sedimenten oder in Wasser aus unmittelbarer Küstennähe nachgewiesen werden (Brandt 1902, Thomsen 1910, Issatschenko 1914, Carey 1938, Vargue \& Brisou 1963). Dabei dürfte es sich meist um die im Erdboden und im Süßwasser verbreiteten Arten Nitrosomonas europaea und Nitrobacter winogradskyi gehandelt haben - also um jene chemoautotrophen Nitrit- und Nitratbakterien, welche die Oxydation von Ammoniak zu Nitrat auf dem Lande besorgen und dabei die Energie zur Kohlensäurereduktion gewinnen. Es wurde daher auch vermutet, daß die 
in den Küstengewässern vorhandenen Nitrifizierer vom Land stammten und sich an die höheren Salzgehalte von Brack- und Meerwasser adaptiert hätten.

Erst vor wenigen Jahren gelang es WATsoN $(1963,1965)$ ein marines, ammoniakoxydierendes Bakterium aus dem Atlantischen Ozean zu isolieren. Bei diesem Nitrosocystis oceanus handelt es sich um ein chemoautotrophes Nitritbakterium, das Ammoniak zu Nitrit oxydiert. Ein Bakterium, das Nitrit zu Nitrat oxydiert, konnte aber in der offenen See bis heute nicht nachgewiesen werden, obwohl es auf Grund der chemischen Befunde dort auch Nitratbakterien geben muß.

In der Ostsee wurden bei früheren Untersuchungen nur in der Kieler Förde bis zu etwa $1 \mathrm{sm}$ Entfernung von der Küste Nitrifizierer im Sediment nachgewiesen, nicht aber im Wasser (Brandt 1902, Thomsen 1910). Auch ist über den Kreislauf des Stickstoffs erst sehr wenig bekannt. Ganz ähnlich liegen die Verhältnisse in der Nordsee. Hier wurden von THOMSEN (1910) in Schlickproben aus der "Fahrrinne südlich Helgoland" lediglich Nitritbakterien gefunden, und LIEBERT (1915) konnte Nitrifizierer nur in Sedimentproben aus unmittelbarer Küstennähe feststellen.

\section{ERGEBNISSE}

Wir haben daher in diesen beiden Meeresgebieten mit Untersuchungen begonnen, die zur Aufklärung der bakteriellen Ammoniak- und Nitritoxydation im Brack- und Meerwasser beitragen sollen. Dazu war zunächst einmal erforderlich, den Jahreslauf der anorganischen Stickstoffverbindungen $\mathrm{zu}$ verfolgen. So wurden bei 2 Stationen in der Kieler Bucht in 1, 5, 10, 15, 20 und $26 \mathrm{~m}$ Tiefe monatliche Bestimmungen des Gehaltes an $\mathrm{NH}_{4}^{+}{ }^{+}, \mathrm{NO}_{2}{ }^{-}$- und $\mathrm{NO}_{3}{ }^{-}$-Stickstoff durchgeführt und das gleiche auch bei weiteren Stationen in größeren Zeitabständen. Die Bestimmung des Ammoniaks erfolgte nach GILlbricht (1961), die von Nitrit und Nitrat nach Grasshoff (1964). Es zeigte sich, daß der Gehalt des Wassers an diesen Verbindungen einem deutlichen Jahresgang unterliegt. Als Beispiel mögen die Ammoniak-, Nitrit- und Nitratstickstoffkurven der bei der Station Breitgrund (Abb. 1) aus $10 \mathrm{~m}$ Tiefe entnommenen Wasserproben dienen (Abb. 2).

Die Nitrit- und Nitratgehalte des Wassers nehmen in den Wintermonaten kräftig zu und erreichen im Februar oder Anfang März ihr Maximum, um dann mit dem Beginn der Phytoplanktonentwicklung - je nach den meteorologischen Verhältnissen zwischen Mitte März und Mitte April - auf einen sehr niedrigen Stand zurückzugehen. Es kommt $z$ war da und dort $z u$ einer voribergehenden sommerlichen Wiederzunahme, die aber stets beträchtlich unter den Wintermaxima bleibt. Ganz anders verhält sich der Ammoniakgehalt des Wassers. Dieser unterliegt zwar im Laufe des Jahres größeren Schwankungen, er scheint aber im Ganzen gesehen im Sommer etwas höher als im Winter zu sein. Der anorganisch gebundene Stickstoff liegt demnach im Sommer zum größeren Teil in Form von Ammoniak, zu Ausgang des Winters dagegen zu 70 bis $90 \%$ als Nitrat vor. Der Verlauf der Nitrit- und der Nitratstickstoffkurven in $A b-$ bildung 2 könnte vermuten lassen, daß die Ammoniakoxydation nur während des Winters erfolgt. Wenn man aber die Werte in den verschiedenen Tiefen vergleicht, sieht man, daß dies nicht zutriff. Denn die erwähnte sommerliche Wiederzunahme des 


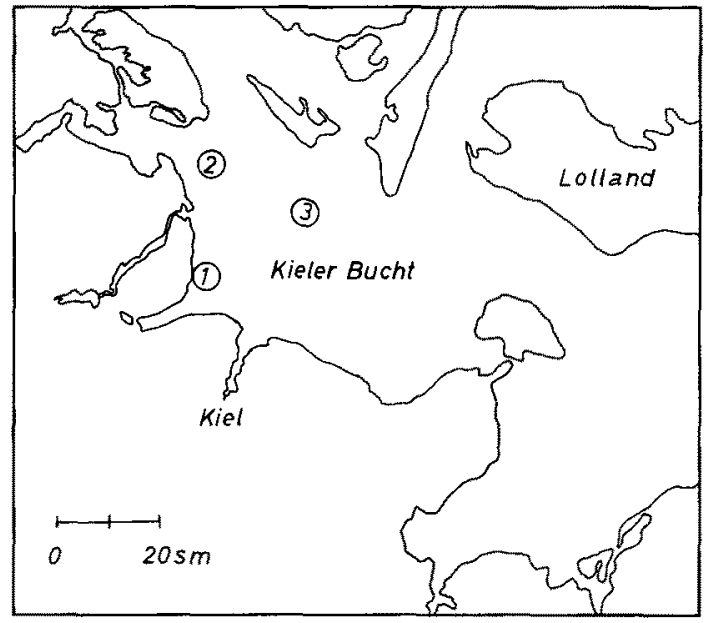

Abb. 1 : Kicler Bucht mit den im Text genannten Stationen: 1 Boknis Eck, 2 Breitgrund, 3 Kieler Bucht Mitte

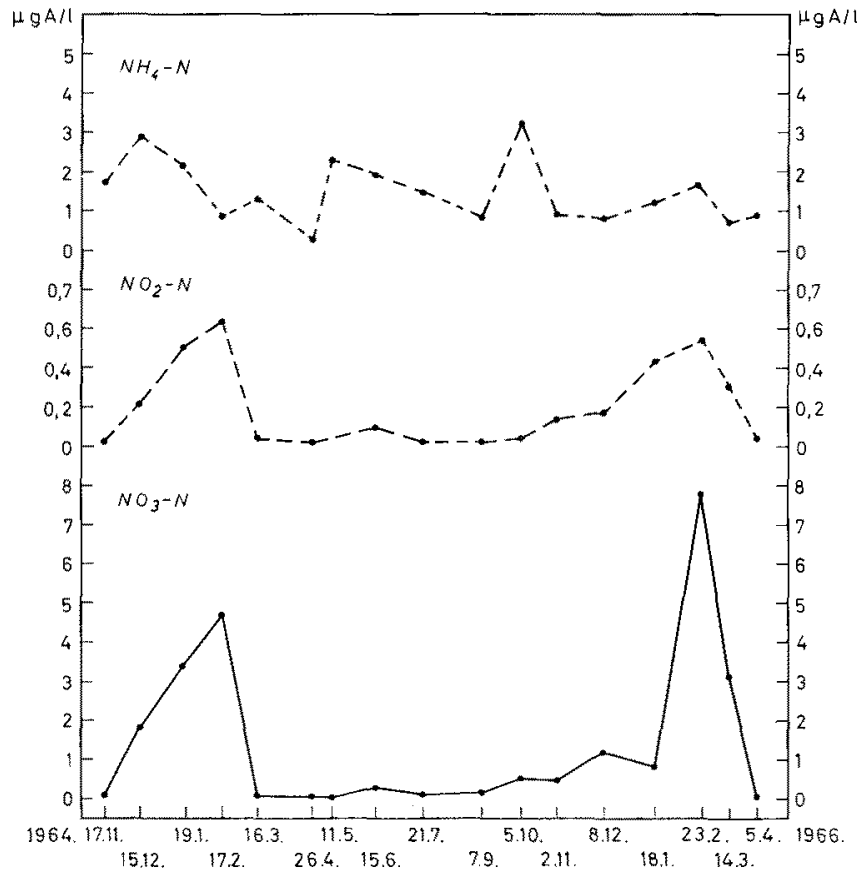

Abb. 2: Ammon-, Nitrit- und Nitratstickstoffgehalte des Ostseewassers bei der Station Breitgrund in $10 \mathrm{~m}$ Tiefe in der Zeit von November 1964 bis April 1966 


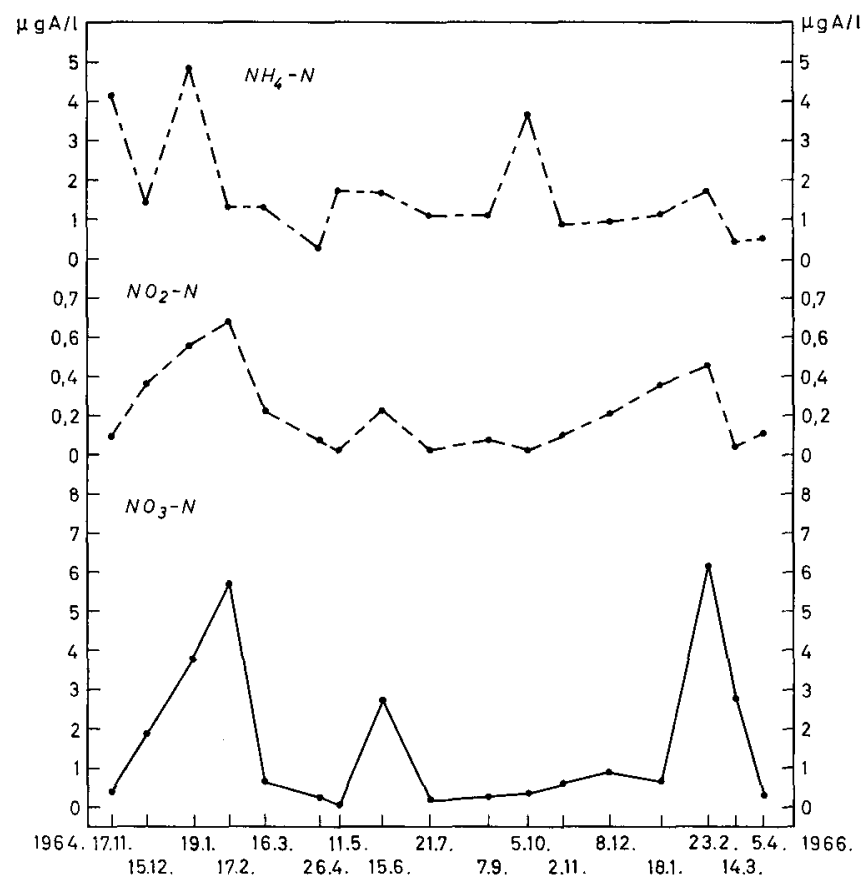

Abb. 3: Ammon-, Nitrit- und Nitratstickstoffgehalte des Ostseewassers bei der Station Breitgrund in $20 \mathrm{~m}$ Tiefe in der Zeit von November 1964 bis April 1966

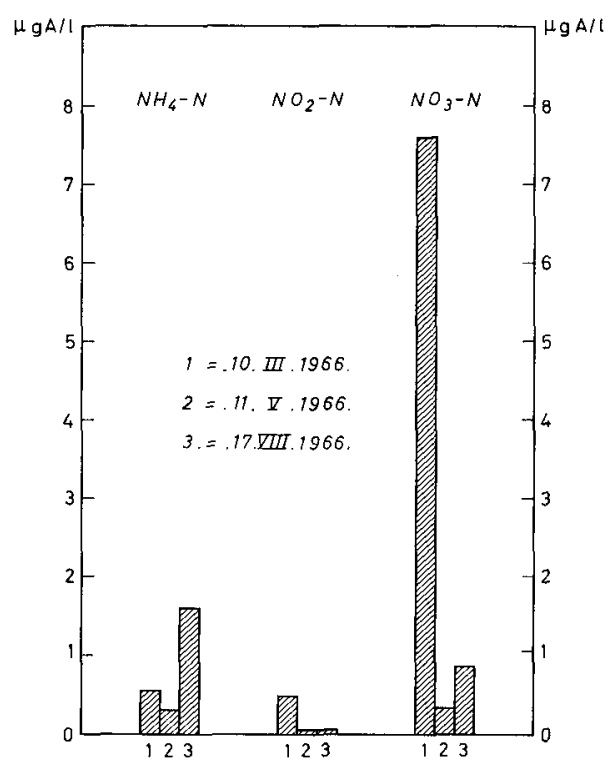

Abb. 4: Ammon-, Nitrit- und Nitratstickstoffgehalte des Ostseewassers bei der Station Kieler Bucht Mitte am 10. März, 11. Mai und 17. August 1966 
Nitratgehaltes ist in den tieferen Wasserzonen oft viel ausgeprägter als in den oberflächennahen Schichten (Abb. 3). Während also zur Zeit des Frühlingsmaximums der Phytoplanktonentwicklung nahezu der gesamte anorganisch gebundene Stickstoff von den Planktonalgen festgelegt ist, wird dann im Laufe des Sommers wieder ein Teil in

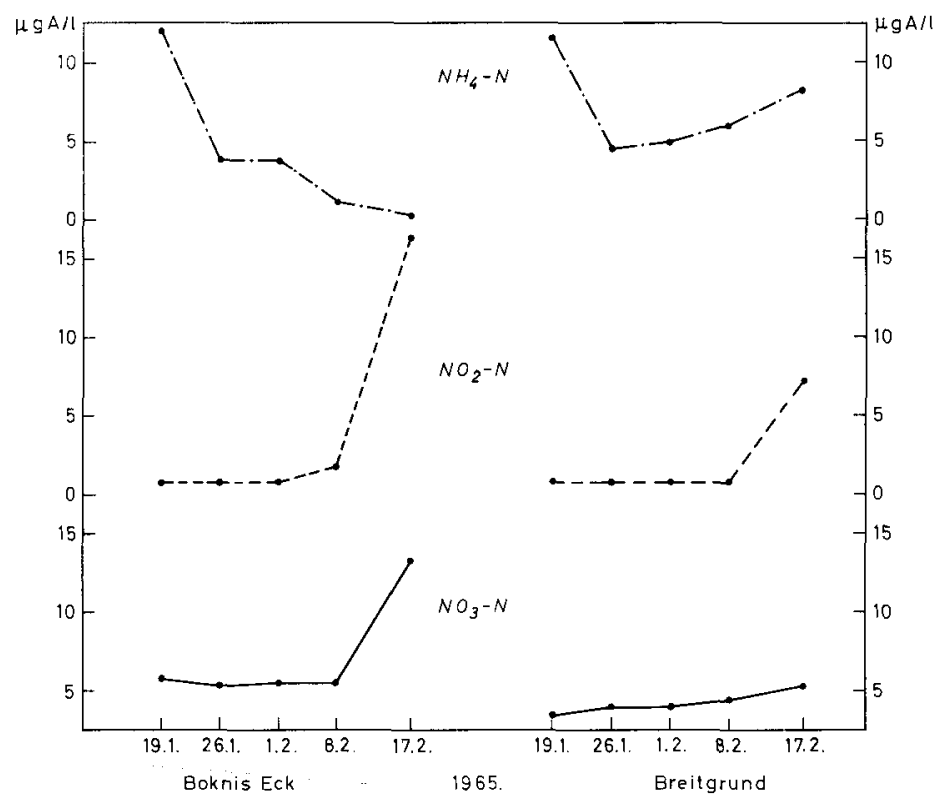

Abb. 5: Ammon-, Nitrit- und Nitratstickstoffkurven von je einer Wasserprobe der Stationen Boknis Eck und Breitgrund ( $1 \mathrm{~m}$ über Grund entnommen) nach Zugabe von $500 \gamma\left(\mathrm{NH}_{4}\right)_{2} \mathrm{SO}_{4}$ im Dunkeln bei Zimmertemperatur. Die Ammoniakgehalte gingen schnell zuruidk und mit einiger Verzögerung stiegen die Nitrit- und Nitratgehalte kräftig an. Das ist unter den Versuchsbedingungen nur durch bakterielle Ammoniakoxydation möglich

Form von Ammoniak freigesetzt, und zwar - bedingt vor allem durch Sedimentationserscheinungen - in den tieferen Zonen $(15-25 \mathrm{~m})$ mehr als nahe der Oberfläche. Das freigesetzte Ammoniak wird dann über Nitrit zu Nitrat oxydiert. Daher steigt in der Regel der Nitratgehalt am stärksten an. Sein Anstieg in der Wasserschicht über Grund ist deshalb besonders groß, weil hier das meiste Ammoniak oxydiert und dabei weniger Stickstoff durch das Phytoplankton verbraucht wird als in der Zone zwischen 5 und $15 \mathrm{~m}$ Tiefe.

Der Jahreslauf der anorganischen Stickstoffverbindungen ist im ganzen Bereich der Kieler Bucht grundsätzlich gleich und zeigt nur verhältnismäßig geringe Unterschiede. So entsprechen die Verhältnisse bei der Station Kieler Bucht Mitte weitgehend denen bei der mehr in Landnähe gelegenen Station Breitgrund (Abb. 4). Der gleiche Jahresgang findet sich auch in den durch Abwässer mehr oder weniger stark verunreinigten Ostseeförden, wie die Untersuchungen von KäNDLER (1963) in der Flensburger Innenförde und eigene Beobachtungen in der Schlei zeigten. Es werden dort natürlich sehr viel höhere Werte erreicht. Dagegen sind die Verhältnisse im Elbe-Ästuar völlig 
anders. Hier nimmt der $\mathrm{NH}_{4}{ }^{+}$-Gehalt in der warmen Jahreszeit stark ab und gleichzeitig kommt es zu einem kräftigen Nitritanstieg infolge der lebhaften $\mathrm{NH}_{4}{ }^{+}-\mathrm{Oxyda}-$ tion durch die Nitritbakterien. Die Ursache für diesen ganz anderen Jahresgang der anorganischen Stickstoffverbindungen im Elbe-Aistuar dürfte in den dort herrschenden völlig anderen hydrographischen, chemischen und biologischen Verhältnissen zu suchen

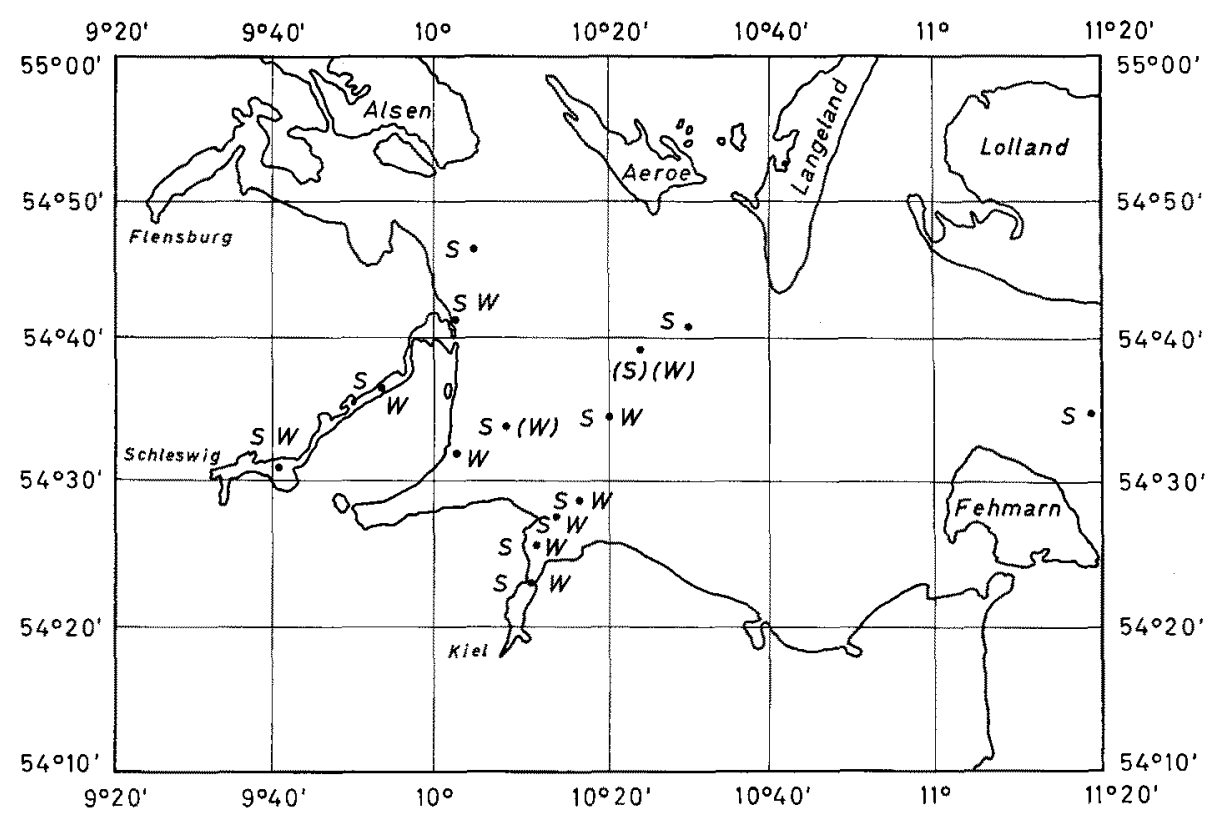

Abb. 6: Skizze der Kieler Bucht mit den Stationen, bei denen nitrifizierende Bakterien nachgewiesen werden konnten: $\mathrm{S}$ im Sediment, W im Wasser $1 \mathrm{~m}$ über Grund; Klammern bedeuten, daß nur Nitritbakterien - nicht aber Nitratbakterien gefunden wurden. (Nach Angaben von $\mathrm{K}$. BANSEMIR)

sein (vgl. RreinHermer 1965). Während in einem Fließgewässer bei niedrigen Wassertemperaturen vor allem die Zeit zu einer nennenswerten Ammoniakoxydation fehlt, spielt in Meeresgebieten der Zeitfaktor im allgemeinen keine Rolle, so daß hier im Laufe des Winters trotz starker Aktivitätsverminderung der nitrifizierenden Bakterien noch eine beträchtliche Ammoniakoxydation möglich wäre.

Um nun festzustellen, ob im Ostseewasser tatsächlich eine durch Bakterien bewirkte Ammoniakoxydation erfolgt, wurden sterilisierte 1-1-Glasflaschen mit $1 \mathrm{~m}$ über Grund entnommenem Wasser von verschiedenen Stationen der Kieler Bucht beschickt, bei Zimmertemperatur dunkel gestellt und darin in einwöchigen Abständen der Gehalt an $\mathrm{NH}_{4}{ }^{+}, \mathrm{NO}_{2}^{-}$und $\mathrm{NO}_{3}^{-}$bestimmt. Es zeigte sich meist sehr schnell eine Abnahme des $\mathrm{NH}_{4}{ }^{+}$-Gehaltes, während - allerdings oft mit einer gewissen Verzögerung - das Nitrat zunahm. Wurde den Wasserproben etwas $\left(\mathrm{NH}_{4}\right)_{2} \mathrm{SO}_{4}(500 \gamma / 1)$ zugesetzt, so nahm das Ammoniak ebenfalls schon in der ersten Woche kräftig ab, der Nitrit- und Nitratgehalt veränderten sich dagegen nur wenig nach oben oder unten. Aber nach 3 bis 4 Wochen 
stiegen sie dann kräftig an, bis schließlich fast der gesamte anorganisch gebundene Stickstoff in Form von Nitrat vorlag. Daraus kann man auf eine bakterielle Ammoniakoxydation in den Wasserproben schließen.

In Abbildung 5 sind die Ergebnisse eines entsprechenden Versuches mit Wasser von den Stationen Boknis Eck und Breitgrund wiedergegeben. In beiden Fällen ging

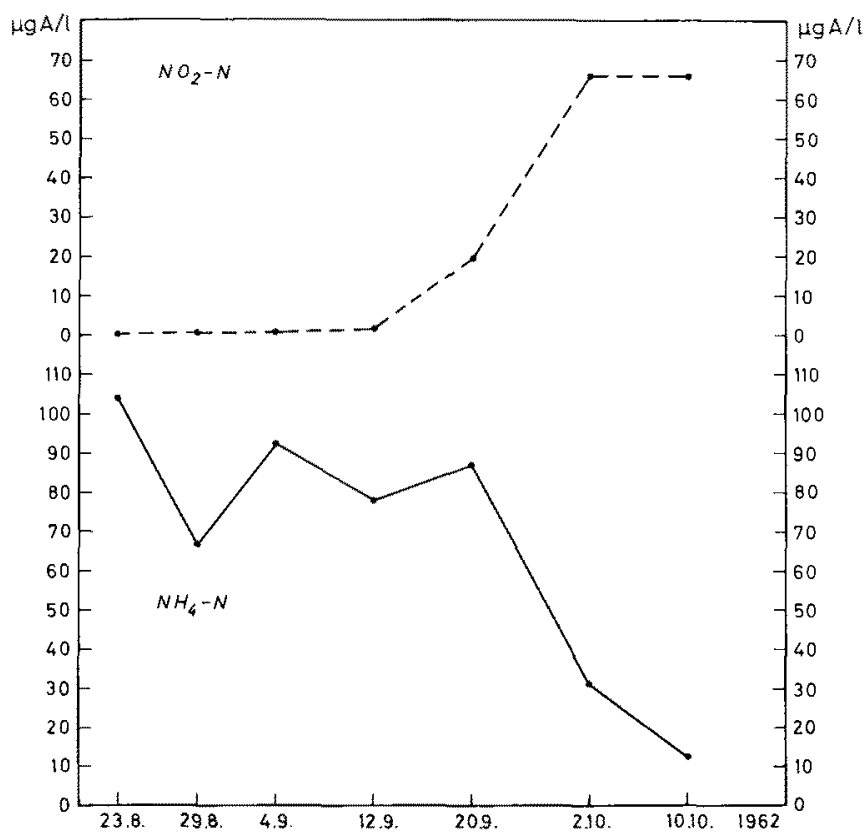

Abb. 7: Ammon- und Nitritstickstoffkurven in einem belüfteten Glasballon mit 201 Nordseewasser nach Zusatz von $96 \mathrm{mg}\left(\mathrm{NH}_{4}\right)_{2} \mathrm{CO}_{3}$ im Dunkeln bei $18^{\circ} \mathrm{C}$. Die starke Abnahme des Ammoniakgehaltes und der kraftige Anstieg des Nitritgehaltes sind auf die Tatigkeit yon Nitritbakterien zurückzuführen

der $\mathrm{NH}_{4}{ }^{+}$-Gehalt in der ersten Woche sehr stark zurück, um dann nur noch langsam weiter abzunehmen oder sogar wieder anzusteigen. In der 3. beziehungsweise 4 . Woche nahmen dann die $\mathrm{NO}_{2}^{-}$- und $\mathrm{NO}_{3}^{-}$-Gehalte kräftig zu. Der starke $\mathrm{NH}_{4}{ }^{+}$-Rückgang zu Beginn des Versuches, der mit keiner entsprechenden Zunahme von Nitrit und Nitrat einherging, ist eine Folge der Ammoniakfestlegung durch heterotrophe Bakterien. Im weiteren Verlauf wird Ammoniak wieder freigesetzt und dann mehr oder weniger schnell über Nitrit zu Nitrat oxydiert. Unter den Versuchsbedingungen kann es sich bei den gefundenen Oxydationsraten nur um eine bakterielle Ammoniakoxydation gehandelt haben.

In der Zeit von Januar bis Juni 1966 wurden dann von K. BANSEMIR Wasser- und Sedimentproben von verschiedenen Stationen der Kieler Bucht auf das Vorhandensein von Nitrit- und Nitratbakterien untersucht (Methodik siehe RHEInHeImer 1965). Es konnten in den meisten Sedimentproben sowohl Nitrit- als auch Nitratbakterien nachgewiesen werden. Das war im allgemeinen auch bei den Wasserproben, die jeweils 
$1 \mathrm{~m}$ über Grund entnommen waren, der Fall (Abb. 6), während in solchen von der Oberfläche nur relativ selten nitrifizierende Bakterien gefunden wurden. Die Oxydationsgeschwindigkeit nahm aber mit zunehmendem Salzgehalt besonders bei den Nitratbakterien $\mathrm{ab}$.

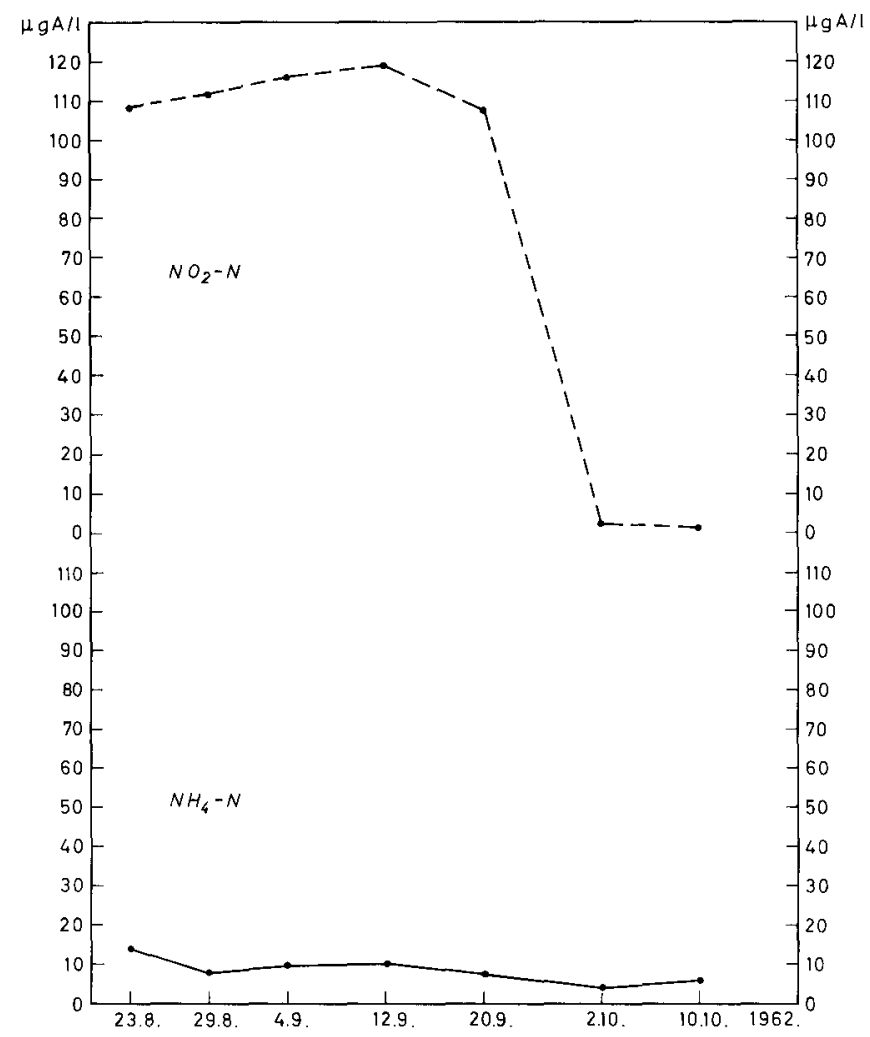

Abb. 8: Ammon- und Nitritstickstoffkurven in einem belüfteten Glasballon mit 201 Nordseewasser nach Zugabe von $138 \mathrm{mg} \mathrm{NaNO} 2$ im Dunkeln bei $18^{\circ} \mathrm{C}$. Die kräftige Abnahme des Nitritgehaltes in der zweiten Hälfte der Versuchszeit ist auf die Tätigkeit von Nitratbakterien zurückzuführen

In den Sommermonaten 1962 und 1963 bei Helgoland durchgeführte Untersuchungen zeigten, daß die Sedimente der tiefen Rinne und weiterer Standorte im Umkreis der Insel ebenfalls Nitrit- und Nitratbakterien enthielten. Das gleiche war auch bei Wasserproben (jeweils 20 l Oberflächenwasser) der Fall, die Ende August 1962 einige Seemeilen westlich von Helgoland entnommen wurden. Nach Zugabe von etwas Ammoniumcarbonat stieg der Nitritgehalt durch die Tätigkeit von Nitritbakterien kräftig an (Abb. 7), und zugesetztes Nitrit verschwand nach einiger Zeit infolge der Oxydation durch Nitratbakterien (Abb. 8).

Im ganzen gesehen nimmt die Zahl der Nitrit- und Nitratbakterien - ebenso wie die Gesamtkeimzahl - mit zunehmender Entfernung von der Küste ab. Das trifft so- 
wohl für die Sedimente als auch für das Wasser zu. Dabei scheinen die Nitratbakterien stärker als die Nitritbakterien abzunehmen. Es sind also im Brackwasser der westlichen Ostsee - ebenso wie im Seewasser der östlichen Nordsee - chemoautotrophe Nitrit- und Nitratbakterien vorhanden, die unter den jeweiligen Salinitätsbedingungen auch zur Ammoniak- beziehungsweise Nitritoxydation befähigt sind. Ob diese aber allein für die Ammoniakoxydation in diesen beiden Meeresteilen verantwortlich sind, läßt sich zur Zeit noch nicht übersehen. Weitere Untersuchungen sollen nun klären, ob es sich bei diesen Nitrit- und Nitratbakterienstämmen in der Nord- und Ostsee lediglich um halotolerante oder (und) um halophile Stämme von Nitrosomonas europaea und Nitrobacter winogradskyi handelt oder ob hier auch noch andere chemoautotrophe Nitrifizierer, wie Nitrosocystis oceanus vorkommen. Ferner muß festgestellt werden, ob neben autotrophen nicht auch heterotrophe Bakterien an der Ammoniakoxydation im Brack- und Meerwasser beteiligt sind.

\section{ZUSAMMENFASSUNG}

1. Seit November 1964 werden in regelmäßiger Folge Bestimmungen des Ammoniak-, Nitrit- und Nitratgehaltes bei verschiedenen Stationen der Kieler Bucht vorgenommen. Es zeigte sich ein deutlicher Jahresgang mit ausgeprägten Nitrit- und Nitratmaxima in der Zeit von Anfang Februar bis Anfang März.

2. Der kräftige Nitratanstieg während des Winters ist - ebenso wie der schwächere sommerliche $\mathrm{NO}_{3}^{-}$-Anstieg - auf die Oxydation von Ammoniak über Nitrit zu Nitrat zurückzuführen. Diese wird im wesentlichen durch Bakterien bewirkt.

3. Dementsprechend konnten im ganzen Bereich der Kieler Bucht und im Seegebiet um Helgoland - sowohl im Wasser als auch in den Sedimenten - chemoautotrophe Nitrit- und Nitratbakterien nachgewiesen werden, die unter den dort gegebenen Salinitätsverhältnissen auch zur Oxydation von Ammoniak beziehungsweise Nitrit in der Lage sind.

Die Untersuchungen wurden von der Deutschen Forschungsgemeinschaft unterstützt. Die Bearbeitung der Nordseeproben erfolgte in der Meeresstation der Biologischen Anstalt Helgoland. Dem Leiter der mikrobiologischen Abteilung der B. A. H., Herrn Dr. Gunkel, und den Herren HaRms und TREKEL möchte ich für ihre wertvolle Hilfe bestens danken. Frau BuCHERT und Fräulein Berg schulde ich Dank für ihre Mitarbeit bei den Ostseeuntersuchungen.

\section{ZITIERTE LITERATUR}

Brandt, K., 1902. Uber den Stoffwechsel im Meere. Wiss. Meeresunters. (Abt. Kiel) 6, $23-79$. CAREY, C. L., 1938. The occurrence and distribution of nitrifying bacteria in the sea. J. mar. Res. 1, 291-304.

ENGEL, H., 1958. Nitrifikation. In: Handbuch der Pflanzenphysiologie. Hrsg. von W. Ruhland (u. a.). Springer, Berlin, Bd 8, 1107-1127.

- 1960. Die Nitrifikanten. In: Handbuch der Pflanzenphysiologie. Hrsg, von W. Ruhland (u. a.). Springer, Berlin, Bd 5, 664-681.

Gillbricht, M., 1961. Eine Methode zum dirckten Nadweis von Ammoniak im Seewasser. Helgoländer wiss. Meeresunters. 8, 58-67. 
GRAsshoff, K., 1964. Zur Bestimmung von Nitrat in Meer- und Trinkwasser. Kieler Meeresforsch. 20, 5-11.

IssatschenKo, B. L., 1914. Investigations on the bacteria of the glacial Arctic ocean (Issledovanija nad bakterijami Severnogo Letovitogo okeana). ([In: Trudy Murmanskoj nauchopromyslovoj ekspedicii 1906. Petrograd, 300 pp.] Zit. nach Zobell 1946.)

KÄNDLER, R., 1963. Hydrographische Untersuchungen in der Flensburger Förde. Kieler Meeresforsch. 19, 142-157.

İEBerT, F., 1915. Uber mikrobiologische Nitrit- und Nitratbildung im Meere. ([Rapp. Verh. Rijksinst. Vissch Onderz. 1, 3.] Zit. nach Zobell 1946.)

RHeinheimer, G., 1965. Mikrobiologische Untersuchungen in der Elbe zwischen Schnackenburg und Cuxhaven. Arch. Hydrobiol. (Suppl. Bd) 29 (2), 181-251.

Thomsen, P., 1910. Ober das Vorkommen von Nitrobakterien im Meere. Wiss. Meeresunters. (Abt. Kiel) 11, 1-27.

Vaccaro, R. F., 1962. The oxydation of ammonia in sea-water. J. Cons. perm. int. Explor. Mer. 27, 3-14.

VARGUE, H. \& BRIsov, J., 1963. Researches on nitrifying bacteria in ocean depths on the coast of Algeria. In: Symposium on marine microbiology. Ed. by C. H. Oppenheimer. C. C. Thomas, Springfield, III., 415-425.

Watson, S. W., 1963. Autotrophic nitrification in the ocean. In: Symposium on marine microbiology. Ed. by C. H. Oppenheimer. C. C. Thomas, Springfield, Ill., 73-84.

- 1965. Characteristics of a marine nitrifying bacterium, Nitrosocystis oceanus sp. n. Limnol. Oceanogr. 10 (Suppl.), R 274-R 289.

Zobell, C. E., 1964. Marine microbiology; a monograph on hydrobacteriology. Chronica Botanica Co., Waltham, Mass., 240 pp.

\section{Diskussion im Anschluß an den Vortrag RHEINHEIMER}

GUNKEL: Soweit mir bekannt ist, arbeitet VrSHNIAC mit anaeroben Bakterien, die $\mathrm{NH}_{4}$ oxydieren, unter Verwendung von $\mathrm{CO}_{2}$ im $\mathrm{O}_{2}$-freien Milieu.

Rhernheimer: Diese Versuche sind mir nicht bekannt.

LÜNEBURG: Wo liegt nach Ihren Erfahrungen das für ein Ästuar typische $\mathrm{NO}_{2}$-Maximum in der Elbmündung?

Rheinhermer: Das sommerliche $\mathrm{NO}_{2}$-Maximum befindet sich in Elbeästuar zwischen Wedel und Glückstadt.

Overbeck: Bleiben Nitrifikanten im anaeroben Wasser längere Zeit lebensfähig, können sie also hieraus isoliert werden?

Rhennhermer: Die nitrifizierenden Bakterien sind noch in einem sehr sauerstoffarmen Substrat lebensfähig; sie scheinen demnach unter anaeroben Bedingungen nicht schnell zugrunde zu gehen. In anaerobem Wasser findet man aber nur selten Nitrifizierer.

OVerbeck : Kann man Nitrifikanten in einem anaeroben, reduzierten Sediment finden?

Rheinheimer: Auch in anaeroben Sedimenten finden sich nur wenig Nitrifizierer; dennoch können sie mitunter daraus isoliert werden. Sie dïrften dann aber aus dem darüber befindlichen aeroben Wasserkörper stammen, da sie sich unter anaeroben Bedingungen nicht entwickeln können.

Overbeck: Was ist über die $\mathrm{H}_{2} \mathrm{~S}$-Toleranz der Nitrifikanten bekannt?

Rheinhejmer: Genauere Untersuchungen liegen meines Wissens darüber nidht vor. Doch dürfte die $\mathrm{H}_{2} \mathrm{~S}$-Toleranz gering sein. 